\title{
CFD Simulations of Two-Phase Gas/non-Newtonian Fluid Flows in Pipes
}

\author{
Miguel A. Daza Gómez ${ }^{1}$, Nicolás Ratkovich ${ }^{1}$, Eduardo Pereyra ${ }^{2}$, Pietro Poesio ${ }^{3}$ \\ ${ }^{1}$ Department of Chemical Engineering, Universidad de los Andes \\ Carrera 1 \# 18a-12, Bogota, Colombia \\ ma.daza299@uniandes.edu.co;n.rios262@uniandes.edu.co \\ ${ }^{2}$ McDougall School of Petroleum Engineering, The University of Tulsa \\ Tulsa, OK 74104, United States \\ eduardo-pereyra@utulsa.edu \\ ${ }^{3}$ Department of Mechanical and Industrial Engineering, University of Brescia \\ Brescia, Italy \\ pietro.poesio@unibs.it
}

\begin{abstract}
Multiphase flow is a well-known studied subject, due to its importance in many industrial processes that affects human kind. This type of flow is found in the upstream of the oil industry and the most frequent pattern is slug flow. This pattern has the characteristic of being an intermittent flow nature that has a sequence of elongated bubbles followed by portions of liquids. Slug flow hydrodynamics is complex and of great importance to be modelled. Many researches have been studying two-phase flow air/water (Newtonian fluid) but in certain occasions the two-phase flow encountered in the industry has the behaviour of non-Newtonian fluids. The objective of this research is the validation of a CFD model with the experimental data obtained in the University of Brescia that represents a two-phase air/non-Newtonian slug flow. The CFD model was implemented with an orthogonal mesh and specific physics that represent the studied system. One of the most significant model is the power-law that describes the behaviour of the nonNewtonian fluid used in this study: CarboxyMethyl Cellulose CMC-water solutions. Three different concentrations of CMC were used $(1,3$ and $6 \% \mathrm{w} / \mathrm{w})$. The parameters evaluated were: slug frequency, dimensionless slug length and pressure drop. The relative error for the first two parameters was $24.22 \%$ and for the pressure drop $45.10 \%$. The overall relative error obtained was of $34.66 \%$. With this results the model is representing the experimental data but it can be improved by slightly changing the used boundary conditions and implementing additional models that can describe in a better way the interface between the two fluids.
\end{abstract}

Keywords: Slug Flow, CFD, Non-Newtonian Liquid, Slug Frequency, Dimensionless Slug Length, Pressure Drop.

\section{Introduction}

The multiphase flow has been a major concern since it is encountered in many industrial processes. One major example of these processes is the flow found in the upstream petroleum industry [1]. The most common multiphase flows is the gas-liquid flow, specifically the slug flow. Slug flow is an intermittent multiphase flow pattern that has a sequence of elongated bullet-shaped bubbles followed by a portion of liquid below the bubble bottom [2]. The hydrodynamics of the flow are rather complex, and its intermittency can introduce a large degree of randomness and instability of the systems where it occurs [2].

This type of flow needs to be thoroughly studied because the knowledge of its fundamentals is necessary to accurately model not only the fluid flow but also reaction and heat and mass transfer in several operation units [2], [3]. Another problem of this type of flow is the possibility to predict a priori the structure based on known values of apparent flow velocity and properties of particular phases and flow geometry (pipe diameter and inclination angle) [4]. The main characteristics are the intermittency and irregularity, and due to its unsteady and developing character, the flow parameters such as the length of the liquid slug and the length of the elongated bubbles should be described [5]. Two important properties are the slug length and the slug frequency, the average slug length has been observed to be about $15-40$ pipe diameters for horizontal flow and 8-25 diameters for vertical flow, and the slug frequency has been considered an entrance phenomena, since it results from the bridging of the liquid at the entrance [5]. 
Non-Newtonian fluids are found in these kind of multiphase flows, especially the shear thinning fluid, as waxy crude oils, gelled oils, drilling muds, fracturing, and slurries [1], [6]. These may be encountered in the extraction of oil and gas and has various problems and challenges due to the behavior they have. The most notable features and characteristics of these are the flow pattern, the phase hold-up and the pressure drop [6]. One of the most widely used model to describe them is the power-law fluid model, which can be applied to a wide range of fluids and conditions but has certain limitations [1]. Deviations of the power law can occur at very low shear rates, and when the fluids are at high shear rates in turbulent flows [1]. One of the first studies that were made of non-Newtonian flow was conducted by Chhabra and Richardson [3] in which they develop a flow pattern based in a Newtonian one.

A useful tool to study and evaluate the hydrodynamics of two-phase air/non-Newtonian fluid flow is Computational Fluid Dynamics (CFD). CFD has the benefit of being capable of changing the operation conditions in a simpler way than in an experimental facility. The conditions that can be changed vary from inclination angles and diameters of the pipes to gas and liquid properties and flow rates. Nevertheless, these studies must be validated with experimental data first, so it can be concluded that the model works and therefore it can be extrapolated.

\section{Methodology}

\subsection{Experimental Facilities}

The experimental data used to validate the CFD simulations was collected at the University of Brescia (Italy) by Picchi [6]. In Fig. 1 it is schematically represented the setup in which the experiments were performed. It is composed by a $9 \mathrm{~m}$ long glass pipe, with an inner diameter of $0.0228 \mathrm{~m} \mathrm{[6].} \mathrm{The} \mathrm{pipe} \mathrm{is} \mathrm{mounted} \mathrm{on} \mathrm{a} \mathrm{rigid} \mathrm{bean} \mathrm{and} \mathrm{it} \mathrm{has} \mathrm{the} \mathrm{option} \mathrm{to}$ change the inclination around the central pin.

The test fluids, that are CarboxyMethyl Cellulose CMC-water solutions, are injected by the injector (letter L in Fig. 1). Air mass flow rate us measured prior to entering the test section by a thermal mass flow meter, and at the same spot, air pressure is monitored. Liquid supply is provided by a centrifugal pump, which draws CMC-water solution stored in a 0.5 $\mathrm{m}^{3}$ tank, and the flow rate is controlled by a frequency inverter. Liquid flow rate is measured with a turbine mass flow meter. A differential pressure transducer is placed $6 \mathrm{~m}$ downstream the injection point and the pressure drop is measured across 1.5 m length [6].

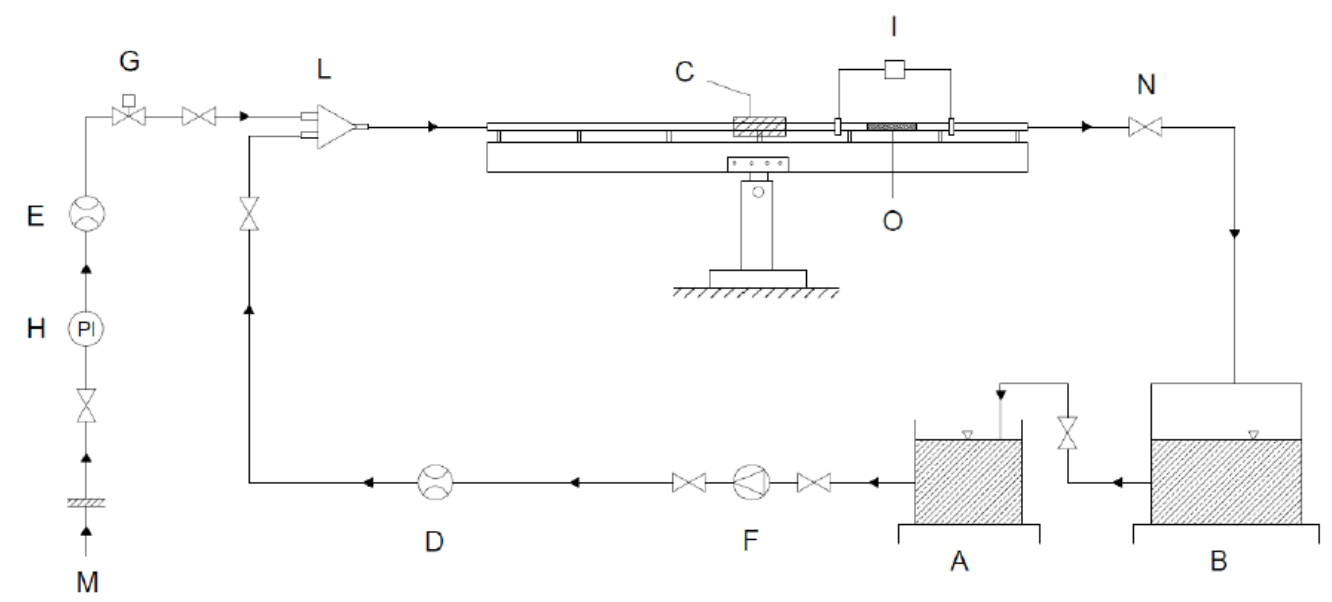

Fig. 1: Sketch of the experimental setup [6]. A-Liquid tank, B-Receiver tank, C-Observation window, D-Turbine flow meter (liquid), E-Thermal mass flow meter (gas), F-Centrifugal pump, G-Valve, H-Pressure transducer, I-Differential pressure transducer, L-Injector, M-Air injection, N-Valve, O-Capacitance probe [6].

Two capacitances sensors (positioned $l_{p}=0.2 \mathrm{~m}$ apart) are placed $7 \mathrm{~m}$ downstream the injection device to characterize slug flows. The pipe-end is at atmospheric pressure and the fluids are discharged into the receiver tank. A glass box is inserted to reduce optical distortions and to allow a correct observation of the flows. A high-speed camera is used to record the flows in the pipe in order to analyze the flow patterns [6]. The average properties of the air are shown in Table 1 and the physical properties of CMC-water solutions are shown in Table 2. The operation conditions of the CMC- 
water solutions (superficial velocity) ranges from 0.05 to $1.4 \mathrm{~m} / \mathrm{s}$ and the gas (superficial velocity) ranges from 0.1 to $2 \mathrm{~m} / \mathrm{s}$ [6].

Table 1: Air properties.

\begin{tabular}{|c|c|}
\hline Property & \\
\hline Viscosity $[\mathrm{Pa} \cdot \mathrm{s}]$ & $1.8 \times 10^{-5}$ \\
\hline Density $\left[\mathrm{kg} / \mathrm{m}^{3}\right]$ & 1.2 \\
\hline
\end{tabular}

Table 2: Physical properties of the test fluids at $25^{\circ} \mathrm{C}$ and atmospheric pressure [6].

\begin{tabular}{|l|c|c|c|c|c|}
\hline & $\begin{array}{c}\text { Conc. } \\
(\boldsymbol{\%})\end{array}$ & $\begin{array}{c}\boldsymbol{\rho} \\
{\left[\mathbf{k g} / \boldsymbol{m}^{\mathbf{3}}\right]}\end{array}$ & $\begin{array}{c}\boldsymbol{m} \\
{\left[\boldsymbol{P a} \cdot \boldsymbol{s}^{\boldsymbol{n}}\right]}\end{array}$ & $\begin{array}{c}\boldsymbol{n} \\
{[-]}\end{array}$ & $\begin{array}{c}\boldsymbol{\sigma} \\
{[\boldsymbol{m} \boldsymbol{N} / \boldsymbol{m}]}\end{array}$ \\
\hline Water & - & $997.5 \pm 0.5$ & $0.001 \pm 0.001$ & 1 & $71.1 \pm 1.8$ \\
\hline CMC-1 & 1 & $998.0 \pm 0.5$ & $0.007 \pm 0.001$ & $0.942 \pm 0.010$ & $72.1 \pm 1.8$ \\
\hline CMC-3 & 3 & $999.0 \pm 0.5$ & $0.061 \pm 0.002$ & $0.875 \pm 0.011$ & $73.7 \pm 1.8$ \\
\hline CMC-6 & 6 & $1002.0 \pm 0.5$ & $0.264 \pm 0.010$ & $0.757 \pm 0.010$ & $76.1 \pm 1.9$ \\
\hline
\end{tabular}

Equation (1) describes the relationship between the shear stress $\left(\vec{\tau}_{y x}\right)$ and shear rate $\left(\overrightarrow{\dot{\gamma}}_{y x}\right)$ for a shear-thinning material.

$$
\left|\vec{\tau}_{y x}\right|=m\left(\left|\overrightarrow{\hat{\gamma}}_{y x}\right|\right)^{n}
$$

\subsection{CFD Modelling}

CFD has been an increasing tool because of its low cost compared to the experimental studies that usually demand sophisticated instruments and large areas, and a different approach may cause a restructuration of the experimental facility that could lead to an even higher cost. A CFD model is proposed to validate and study the experimental results of Picchi [6]. The CFD simulations are developed in the commercial software STAR-CCM+ v12.04 (Siemens, Germany).

The geometrical domain is the first thing to do, due to its importance of being the computational domain. The experimental facility that Picchi uses is a $9 \mathrm{~m}$ and $0.0228 \mathrm{~m}$ ID glass pipe. This pipe is horizontal and has two slight inclinations $\left(5^{\circ},-5^{\circ}\right)$ to give three combinations. These geometries are designed using Autodesk Inventor $®$ v2016 and transformed into CAD models.

Grid generation is a crucial step for the validation of this research project. The mesh arrangements that exist are multiple but for the modelling of multiphase flow in a pipe the best arrangement is the orthogonal grid (also known as "butterfly grid") [7]. An example of this type of grid was made by the operation of direct mesh tool of the program STAR$\mathrm{CCM}+$. Only half pipe is used in the spatial discretization due to the assumption of axial symmetry in the pipe and that it has the advantage of decreasing the computational time.

The specification of the boundary and initial conditions of the system is an important step in the pre-processing stage in CFD. The inlet and outlet of the spatial discretization (pipe) are modelled as velocity inlet and pressure outlet, respectively. The symmetry as a symmetry plane and the pipe wall as wall.

Another important is step the physics model selection. There are two ways to describe the multiphase flow in a pipe: the Eulerian - Eulerian model and the Lagrangian - Eulerian model. The first one treats the fluid phases as interpenetrating continua and study their dynamics by the means of averaged equations of motion [8]. On the other hand, the second model tracks the motion of each particle and solve the dynamics of the fluid at a length smaller than the particle diameter [8]. The last one is chosen, specifically the Volume of Fluid (VOF) model. This model is capable of simulating flows of several immiscible fluids and resolving the interface between the phases of the mixture. It uses only one set of equations for the continuous phase, and the dispersed phase has an additional equation for its volume fraction [9]. The additional models used are: unsteady model, $k-\omega$ turbulence model, segregated flow model, and gravity model. 
The VOF model refers to the flow and the interaction between the distinct phases in the system in which different interfaces between them exist. It simulates the flow of various immiscible fluids and the phase distribution of each phase. An only set of equations of moment are shared by the fluids and the volume fraction is tracked through the computational cell domain. This model also assumes that the phases of the multiple fluids share velocity, pressure and temperature fields. The continuity equation, which guarantees the conservation of mass is described in equation (2).

$$
\frac{\partial \rho}{\partial t}+\frac{\partial \rho v_{i}}{\partial x_{i}}=0
$$

Where $v_{i}$ is the fluid velocity, $t$ is the time, and $x_{i}$ is the spatial coordinate. In equation (3) the momentum is described.

$$
\frac{\partial}{\partial t}\left(\rho v_{j}\right)+\frac{\partial}{\partial x_{i}}\left(\rho v_{i} v_{j}\right)=-\frac{\partial P}{\partial x_{j}}+\frac{\partial}{\partial x_{i}} \mu\left(\frac{\partial v_{i}}{\partial x_{j}}+\frac{\partial v_{j}}{\partial x_{i}}\right)+\rho g_{j}+F_{j}
$$

In this equation, $F, P$, and $g$ indicate external force per unit volume, pressure, and gravitational acceleration respectively, $x_{j}$ is the spatial coordinate, $\mu$ is the dynamic viscosity, and $v_{j}$ is the fluid velocity. The properties of the fluid are calculated as function of the physical properties of each phase and their void fractions.

$$
\begin{aligned}
& \rho_{m}=\sum_{i=1}^{n} \alpha_{p} * \rho_{p} \\
& \mu_{m}=\sum_{i=1}^{n} \alpha_{p} * \mu_{p}
\end{aligned}
$$

Where $\rho_{p}$ is the density of the phase $p$ and $\mu_{p}$ is the dynamic viscosity of the phase $p$. The variable $\alpha_{p}$ is the void fraction and can be expressed as equation (5).

$$
\frac{\partial \alpha_{p}}{\partial t}+u \nabla\left(\alpha_{i}\right)=0
$$

To validate the CFD model the simulations must be transient. The necessary parameters specified in the model are time step, inner iterations per time step and maximum physical time. The most important parameter is the time step, because, if it is not correctly calculated, diverse problems can appear. One of these problems is convergence, which presents when the time step is larger than the velocity magnitude. This causes that intermediate points are not solved, producing that the next points have no previous solution and the CFD solver assuming those solutions that lead to divergence. The CFL (Courant - Friedrichs - Lewy) condition is used to avoid the divergence problem. The recommended CFL values are below 0.1 to capture accurately the interface.

The main results that are obtained from the simulations are: pressure drop, slug frequency, length, and velocity. These results are retrieved from the positions where the capacitance probes are located as explained in section 2.1. The validation of the CFD simulations are of great importance, therefore, it can be proved they can be reliable. The superficial velocities that were used to compare the experimental results are shown in Table 3 . These conditions are simulated in the horizontal pipe with no inclination. With these different cases it is possible to conclude that CFD has the capacity to reproduce the flow pattern encounter on the pipe. 
Table 3: Used cases to validate the CFD simulations.

\begin{tabular}{|c|c|c|}
\hline Fluids & $\begin{array}{c}\mathbf{U}_{\mathrm{SL}} \\
{[\mathbf{m} / \mathbf{s}]}\end{array}$ & $\begin{array}{c}\mathbf{U}_{\mathrm{SG}} \\
{[\mathbf{m} / \mathbf{s}]}\end{array}$ \\
\hline \multirow{3}{*}{ CMC-1 } & 0.95 & \multirow{3}{*}{1.22} \\
\hline & 0.72 & \\
\hline & 1.18 & \\
\hline \multirow{3}{*}{ CMC-3 } & 1.06 & \multirow{3}{*}{1.50} \\
\hline & 0.83 & \\
\hline & 1.29 & \\
\hline \multirow{3}{*}{ CMC-6 } & 0.62 & \multirow{3}{*}{1.50} \\
\hline & 0.45 & \\
\hline & 0.83 & \\
\hline
\end{tabular}

\section{Results and Discussion}

\subsection{Solution Appearance}

In Fig. 2 it is shown the visualization of the modeled slug flow. These images are from the first meters of the pipe. It can be seen the formation of the bubble and the where it has its end. A different approach of visualization is seen in Fig. 3, this time it is observed the volume fraction of the air. The slug pattern does not has the expected visualization because of the interface created by the orthogonal mesh near the wall. This mesh has the problem of creating an elongated cell that could not recreate in the best way the interface between the CMC and the air.

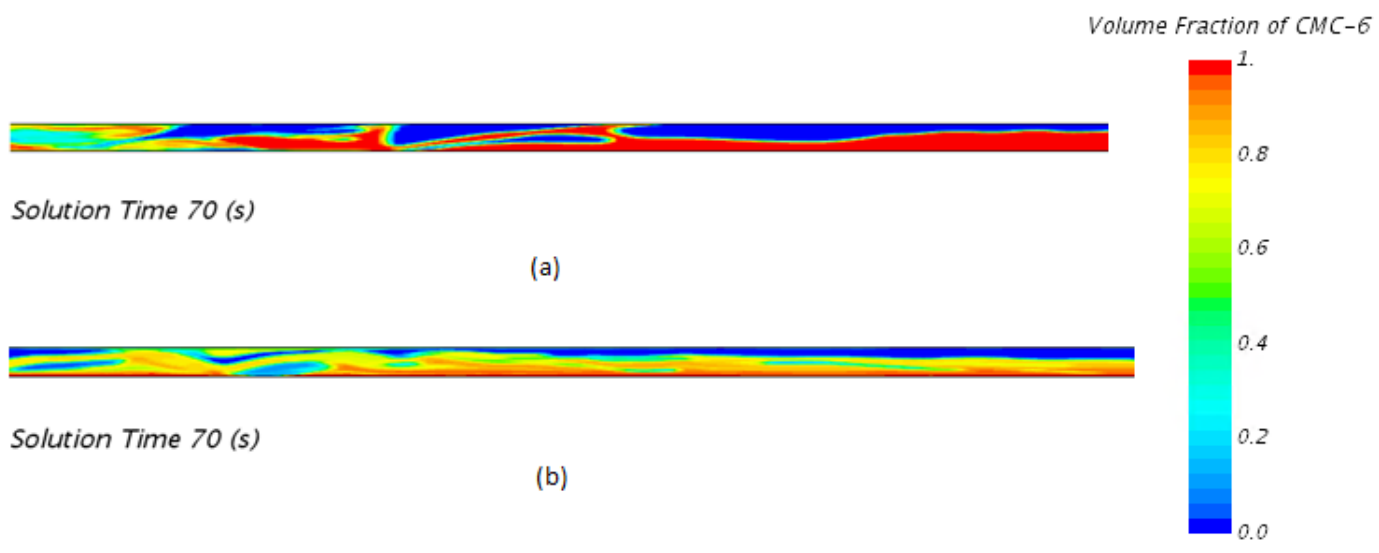

Fig. 2: Visualization of the volume fraction of CMC 6 (a) first meters of the pipe and (b) last meters of the pipe. 


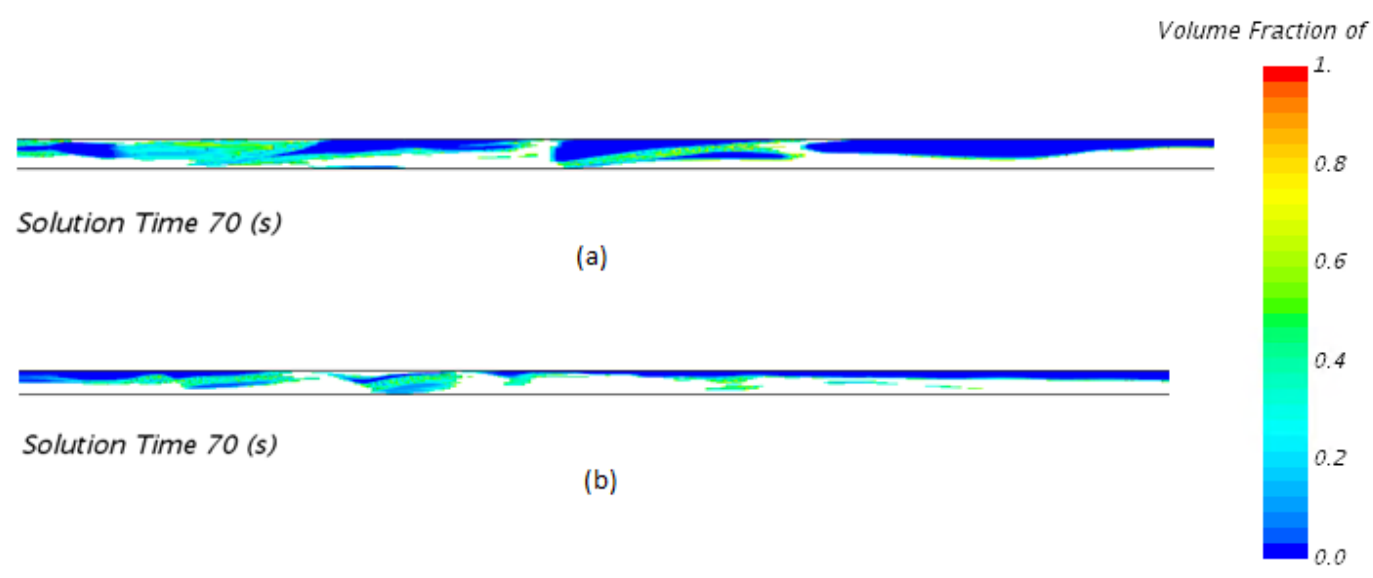

Fig. 3: Different approach of visualization. Volume fraction of air (a) first meters of the pipe and (b) last meters of the pipe.

\subsection{Grid Independence Test}

The grid independence test was done with the CMC-6 fluid. The variation made in the cells was in the axial direction. The starting point was with 1184 axial divisions that lead to 823,918 cells and it was incremented 50 and $70 \%$ in the axial divisions to get 1776 divisions - 1,236,096 cells and 2013 divisions - 1,400,909 cells, respectively.

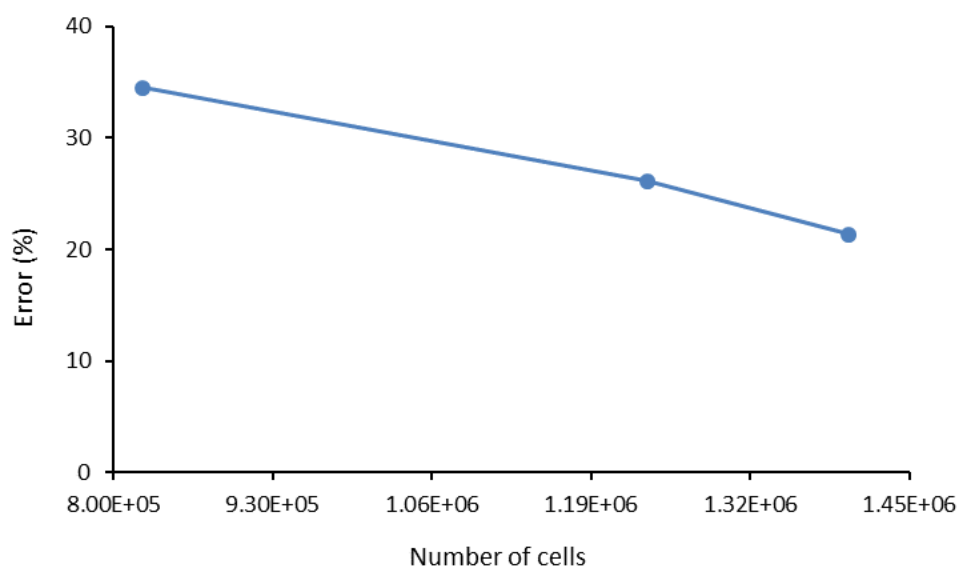

Fig. 4: Grid independency test.

In Fig. 4 a comparison between the three meshes is shown. The comparison was made with the value of the pressure drop. The error decreases when the number of cells increases but the computational time increases significantly. With these results it was decided to choose mesh 2 to develop the rest of the project. Nonetheless the other parameters (slug frequency, length and units) will be compared to achieve a better grid independence test in a future work.

\subsection{Slug Frequency and Length}

The slug frequency and length were compared to the experimental results of Picchi [6] by the means of a Matlab® code. This code processes the signals of the sections where the probes are, by a threshold technique. This technique consists in obtaining equivalent rectangular waves from the probe signals (in the case of CFD, the planes where the information is being acquired). The results of the frequency slug and dimensionless slug length for three different mix velocities meant for the CMC-6, CMC-3, and CMC-1 fluids are shown in Fig. 5. These plots present 4 points that are outside the $\pm 30 \%$ acceptable region. This could be a consequence of the boundary conditions implemented in the model. The average relative error of the CFD model for this two parameters is $24.22 \%$. 


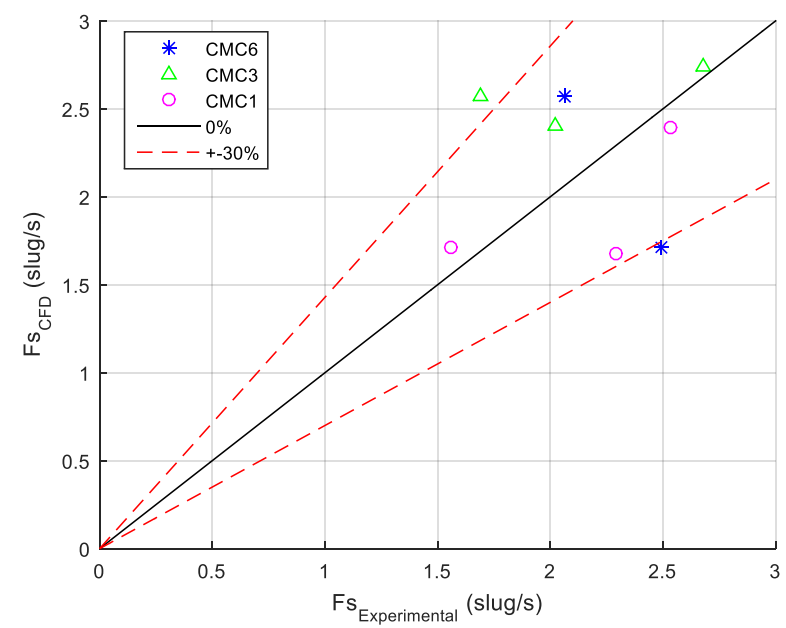

(a)

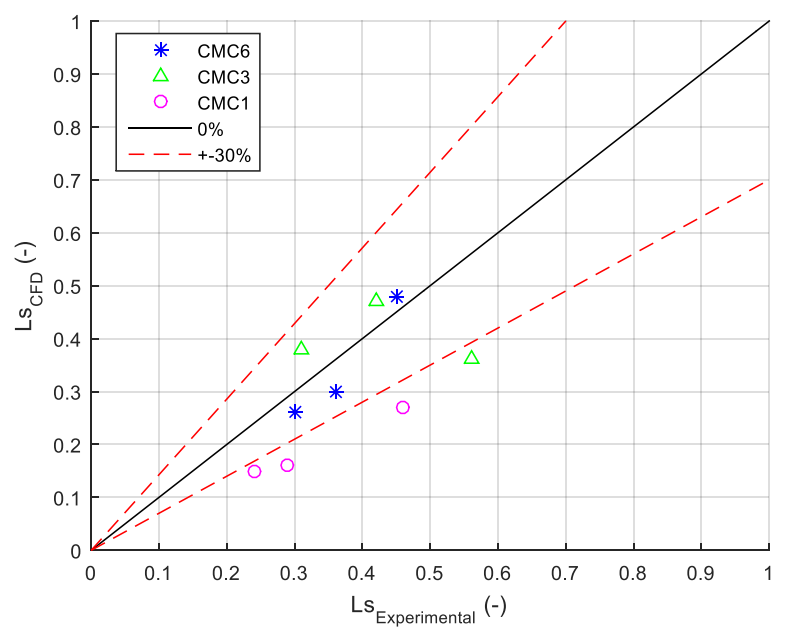

(b)

Fig. 5: Comparison between experimental and CFD results (a) Frequency slug (b) Dimensionless slug length.

\subsection{Pressure Drop}

Another valuable result is the comparison of the pressure drop between the experimental data and the CFD model. The boundary conditions used in the CFD model is over predicting the pressure drop in the case of the CMC-3 fluid as it can be seen in Fig. 6. The overall average relative error is $45.10 \%$ and $25.67 \%$ discarding the CMC-3 fluid. Nevertheless, the model is capable of representing the experimental data and could be used to test other conditions and configurations with a slight modification of the boundary conditions so it could be more precise.

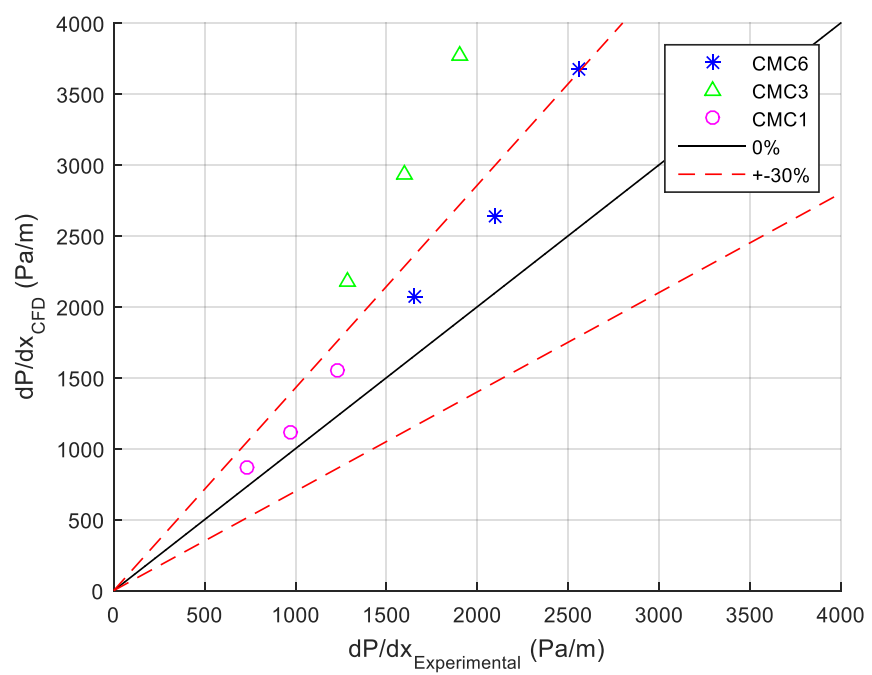

Fig. 6: Comparison between experimental and CFD results of pressure drop.

\section{Conclusion}

The proposed CFD model has the capability to represent the experimental data obtained by Picchi [6]. This is demonstrated by the results of slug frequency, dimensionless slug length, and pressure drop. Although there are a number of points that do not achieve the goal of being in the $\pm 30 \%$ error, the global relative error of the whole CFD model is $34.66 \%$. This means that the mode can be improved with the right changes to make it more strong and confident. To improve the model, a series of changes in the boundary conditions have to be implemented for example the initialization 
could be that air is already inside the pipe and then fluid enters or vice versa, as well as using certain models that can enhance the interface of the two phases that could be the Interface Momentum Dissipation and the RSM turbulence After this is accomplished the model will be extrapolated to different pipes configurations. One of the ideas is to prove it in risers and analyze the severe slugging that is present in this type of configurations.

\section{References}

[1] R. D. Kaminsky and S. Flow, "Predicting Single-Pliase and Two-Phase Non-Newtonian Flow Beliavior in Pipes," vol. 120, no. March 1998, 2016.

[2] J. D. P. Araújo, J. M. Miranda, and J. B. L. M. Campos, "CFD Study of the Hydrodynamics of Slug Flow Systems: Interaction between Consecutive Taylor Bubbles," Int. J. Chem. React. Eng., vol. 13, no. 4, pp. 541-549, 2015.

[3] R. P. Chhabra and J. F. Richardson, "Prediction of flow pattern for the co-current flow of gas and non-newtonian liquid in horizontal pipes," Can. J. Chem. Eng., vol. 62, no. 4, pp. 449-454, 1984.

[4] M. Dziubinski, H. Fidos, and M. Sosno, "The flow pattern map of a two-phase non-Newtonian liquid-gas flow in the vertical pipe," Int. J. Multiph. Flow, vol. 30, no. 6, pp. 551-563, 2004.

[5] D. Barnea and Y. Taitel, "A model for slug length distribution in gas-liquid slug flow," Int. J. Multiph. Flow, vol. 19, no. 5, pp. 829-838, 1993.

[6] D. Picchi, "Theoretical and Experimental Investigation of Two-Phase Gas/non-Newtonian Fluid Flows in Pipes," University of Brescia, 2015.

[7] V. Hernandez-Perez, M. Abdulkadir, and B. J. Azzopardi, "Grid Generation Issues in the CFD Modelling of TwoPhase Flow in a Pipe," J. Comput. Multiph. Flows, vol. 3, no. 1, pp. 13-26, 2011.

[8] L. Mazzei, "Eulerian modeling and computational fluid dynamics simulation of mono and polydisperse fluidized suspensions," University College London, 2008.

[9] Siemens, "STAR-CCM+ DOCUMENTATION." NY, 2015. 